\title{
8. Calcium Concentration Changes in the Aesthetasc Hairs Caused by the Environmental Salinity Shifts
}

\author{
By Shiko CHICHIBU*) \\ Department of Physiology, Kinki University \\ School of Medicine, Sayama, Osaka \\ (Communicated by Yasuji Katsuki, M. J. A., Jan. 16, 1979)
}

It has been known that the aesthetasc hairs on the crustacean antenna serve as the chemoreceptor, ${ }^{6), 9)}$ They are distributed on the external flagellum with their characteristic morphological features distinctive from other setae which have the mechanoreceptive functions..$^{3,44,6), 9), 10)}$

The aesthetasc hairs of the crayfish, Procambarus clarkii, are distributed on the distal two-thirds of the external flagellum.1),3) They are forming two separate hair-tufts on each antennal segment; the distally-located hair-tuft is commonly furnished with guard hairs on both sides. The aesthetasc hair located on the proximal side is usually short and no guard hair is observed. Though the size of the proximallylocated hairs are small, there is no obvious histological difference among them. ${ }^{1), 2)}$ The crayfish aesthetasc hair is divided into three portions from their structural differences $;^{1), 3}$ ) the distal ( $d$ in Fig. $1 \mathrm{~A})$, the middle $(\mathrm{m})$ and the proximal $(\mathrm{p})$ portions. The lumen of the hair is communicating to the surrounding medium through an apical pore, and the interior of the distal portion is apparently homogeneous without rigid structure. Several dyes, such as methylene blue and crystal violet, are easily penetrable into this portion. ${ }^{1)}$ On the contrary, the middle and the proximal portions are filled with tissues, so that dyes can not penetrate into these portions. The tissue elements in these portions are containing neural elements which have the function of chemical transduction. ${ }^{4,7)}$

Application of several different histochemical techniques for divalent alkaline earth metals revealed the localization of calcium and magnesium within the lumen of the crayfish aesthetasc hairs. ${ }^{1,2)}$ The localization of calcium is mainly in the proximal portion with a weaker distribution in the middle portion. On the other hand, the localization of magnesium is limited to the boundary zone between the distal and the middle portions and to the very basal part of the proximal portion.

*) Mailing address: Department of Physiology, Kinki Univ. Sch. Med., Nishiyama 380, Sayama-cho, Osaka 589, Japan. 
Though the precise function of these metals are not yet clear, it was observed that the amount of calcium in the middle and the proximal portion which was measured histochemically, changed considerably by the acclimatization processes to higher osmotic saline solutions of various salinity. In order to study the relationship between the environmental salinity and the amount of calcium inside of the aesthetasc hair lumen, the colorimetric method with microscopic spectrophotometry was used. ${ }^{2), 3)}$

The histochemical reaction used was the von Kossa's silver nitrate transition method. ${ }^{8)}$ Because that the reaction also gave positive coloration for other ions, the resulted dark spot in the aesthetasc hair was checked simultaneously with other methods for calcium. ${ }^{1-3)}$ The stained spot with the von Kossa's method gave good parallelism to those by other methods, including the Dahl's alizarin S method and the Okamoto-Senoo-Kato's direct and indirect methods. ${ }^{8)}$ The von Kossa's method produced reddish-brown black spot for calcium deposit. In order to get intermediate optical density for spectrophotometry, the following modification was made for convenience: The specimen was put into a Petri dish containing $1 \%$ silver nitrate solution, and irradiated with a tungsten lamp for $40 \mathrm{~min}$. The illumination intensity was adjusted between $4.8-5.0 \times 10^{3}$ lux. After washing with distilled water, the preparation was soaked in $2 \%$ sodium thiosulfate solution for $15 \mathrm{~min}$.

The colorimetry was made with a microscopic spectrophotometer (DMSP II, Olympus Optical Co., Tokyo) on the wavelength range between 400 and $700 \mathrm{~nm}$. The spectral transmittance curves thus obtained were processed for chromaticity co-ordinate calculations. When a flagellum was stained, the most of the aesthetasc hairs were stained similarly, but instead of the similar tendency there observed a variety of staining tone between hairs. From this reason, the spectrophotometric measurements were carried out on typically stained hairs selectively. The diameter of the monochromatic spot used was $5.3 \mu \mathrm{m}$ uniformly, and this spot size was satisfactory for passing through the hair lumen freely. The average brightness was indicated by $\mathrm{Y}^{\prime}$ value in the $\mathrm{x}, \mathrm{y}$ and $\mathrm{Y}^{\prime}$ chromaticity co-ordinate system.

The saline solutions used for acclimatization were of two different compositions: Schmalz's artificial sea water (ASW) ${ }^{5)} ; \mathrm{NaCl} 482, \mathrm{KCl}$ $9, \mathrm{MgCl}_{2} 27, \mathrm{MgSO}_{4} 28, \mathrm{CaCl}_{2} 11$ (in $\mathrm{mM}$ ). The total ionic concentration was $1152 \mathrm{mM}$. Van Harreveld solution (v.H.) ; $\mathrm{NaCl} 205, \mathrm{CaCl}_{2}$ $14, \mathrm{KCl} 5, \mathrm{MgCl}_{2} 3, \mathrm{NaHCO}_{3} 2$ (in $\mathrm{mM}$ ). The total ionic concentration was $474 \mathrm{mM}$. Dilutions were made by mixing distilled water with these solutions. In order to decrease free calcium ions in the environment, solutions containing $5 \mathrm{mM}$ EDTA sodium salt and $10 \mathrm{mM}$ citric 

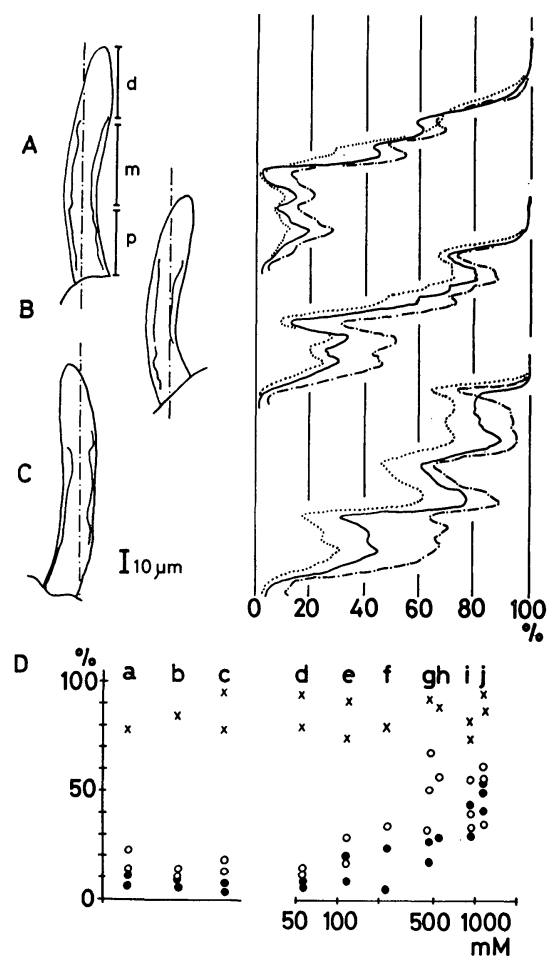

Fig. 1. A-C: Examples of monochromatic brightness changes along the axis of the aesthetasc hairs stained with the von Kossa's method. A, control; B, $1 / 4 \times$ v.H.; C, $3 / 4 \times \mathrm{ASW}$. In each set of curves, the continuous line indicates the transmittance change at $540 \mathrm{~nm}$, the dot-line at $450 \mathrm{~nm}$, and the broken line at $650 \mathrm{~nm}$, respectively. Inset drawings were traced after photographs, and the scanned lines were determined from pictures which were taken before and after the scannings. D: Average brightness (ordinate) versus total ionic concentrations in $\mathrm{mM}$ (abscissa). Acclimatized solutions were as follows: a, $10 \mathrm{mM}$ citric acid; b, $5 \mathrm{mM}$ EDTA-Na salt; c, control with tap water; d, $1 / 8 \times$ v.H.; e, $1 / 4 \times$ v.H.; f, $1 / 2 \times$ v.H.; g, $1 \times$ v.H.; h, $1 / 2 \times$ ASW ; i, $2 \times$ v.H.; j, $1 \times$ ASW. Crosses are the average brightness values of the distal portion, open circles at the middle portion and filled ones at the proximal portion.

acid, respectively, were used. The acclimatization of crayfish to saline solutions of various salinity was made for at least three days before measurements.

The results shown in Fig. 1, A-C illustrated the curves of transmittance changes along the hair axis. As the localization of calcium became reddish-brown black spot, the transmittance curves generally tended to give larger transmittance for red-light spot. The distal portion was almost colorless and transparent. But in the middle and the proximal portions, the discrepancies between curves due to the 

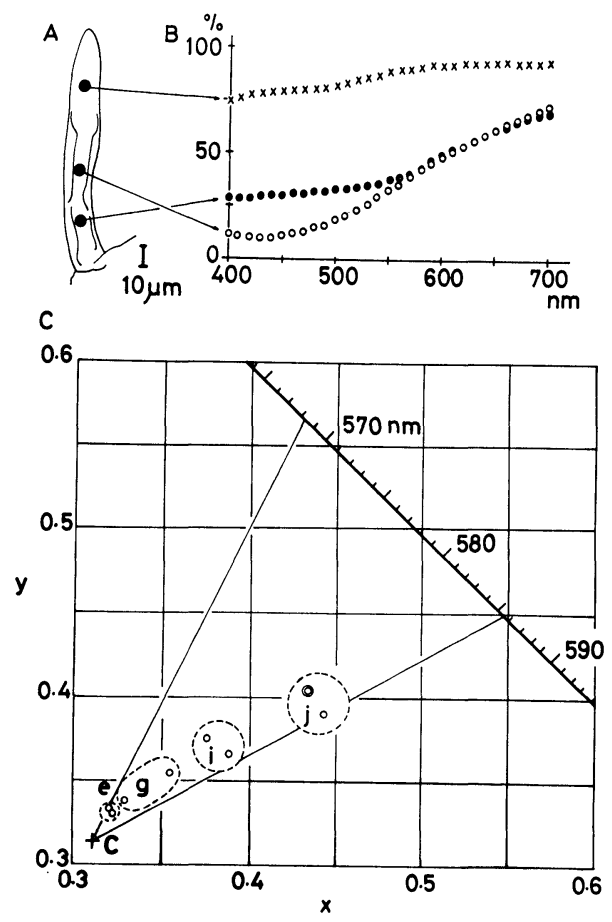

Fig. 2. A and B: Spectral transmittance curves in three different loci in an aesthetasc hair from an ASW-treated crayfish. Note larger transmittance increases in the red-light region both at the middle and the proximal portions. The filled circles in the inset show the relative size and the measured locations of the monochromatic spot to that of the hair. C: Chromaticity co-ordinates collected from the middle portion of aesthetasc hairs. The alphabetical letters, e.g. $i$ and $j$ indicate ionic concentrations of the acclimatized solutions similar to those in Fig. 1D. A cross marked by $\mathrm{C}$ indicates the coordinate of the white light. The double open circle in $j$ is the one obtained from the middle portion of the hair shown in Fig. 2, A and $\mathrm{B}$.

increase of coloration were appeared as well as the transmittance decrease. The comparison of these curves showed the increased transmittance in preparations from crayfishes acclimatized to the higher salinity, and also the increase was distinct to the red light. Fig. 1D illustrated the distribution of the average brightness in each portion of hairs in relation to the acclimatized salinity. The average brightness denoted by $\mathrm{Y}^{\prime}$ value in the proximal portion was smaller than that in the middle portion generally, so that the apparent color in the proximal portion was deeper than that in the middle portion instead of the similar $\mathrm{x}$ and $\mathrm{y}$ values in the chromaticity co-ordinate system. The control experiments with citric acid and with EDTA sodium also gave the similar tendency. 
When the environmental medium was replaced with high-salinity solutions over $120 \mathrm{mM}$ concentration $\left(1 / 4 \times \mathrm{v}\right.$.H.) the $\mathrm{Y}^{\prime}$ values both in the middle and the proximal portion showed a trend of gradual increase. This tendency became clear when the total ionic concentration of the medium reached up to the crayfish's physiological saline solution $(1 \times v . H$. $)$. In the higher concentration range, the average brightness of these portions increased in proportion to the environmental ionic concentration. With this process, the color of these portions gradually added reddish tone.

As the optical density of a color is apparently parallel to the localized calcium concentration, ${ }^{2), 3)}$ it is deducible that the calcium concentration surveyed by the von Kossa's method both in the middle and the proximal portions decreased in the high-salinity environment.

A set of spectral transmittance curves obtained at a certain locus over the wavelength of the visible light was shown in Fig. 2, A and B, and this was measured on the aesthetasc hair from an ASW-treated crayfish. As seen in this figure, the color in the middle portion showed a smaller transmittance value in the blue-green region in comparison to that in the proximal portion. This difference gave stronger reddish tone in the middle portion. In the control without high salinity medium, the corresponding transmittance curves showed smaller values as well as the small discrepancies all the way through the visible light wavelength. The spectral transmittance curves of the distal portion showed little change, and this gave the impression of colorlessness.

The diagram shown in Fig. $2 \mathrm{C}$ is a part of a chromaticity diagram based on the $\mathrm{x}, \mathrm{y}$ and $\mathrm{Y}^{\prime}$ co-ordinate system, and the circles which were denoted by $\mathrm{x}^{\prime} \mathrm{s}$ and $\mathrm{y}^{\prime} \mathrm{s}$ only were plotted by ignoring $\mathrm{Y}^{\prime}$ values. When the chromaticity co-ordinates were calculated from spectral curves, a color was represented by $\mathrm{x}$ and $\mathrm{y}$ on a plane of the color space with $\mathrm{Y}^{\prime}$ which was the representative index of the brightness. In this figure, the chromaticity co-ordinates of these microscopically-measured colors were compiled by only $\mathrm{x}$ and $\mathrm{y}$ co-ordinates for convenience, therefore this procedure ignored the brightness of the colors. These circles were the projections of each color having different $\mathrm{Y}^{\prime}$ value to the plane where $\mathrm{Y}^{\prime}$ was $100 \%$. Each group was in the gamut of red though scattered, and arranged nearly on a line as a whole.

The dominant wavelength of a color is obtainable by combining the co-ordinate to that of the white light, and then extend the line to the periphery of the diagram until the margin of the chromaticity plane. The dominant wavelength thus obtained in these colors in the middle portion from crayfishes treated with various salinity ranged from 568 to $586 \mathrm{~nm}$. With these scattered distribution of the chro- 
maticity co-ordinates, it is difficult to find out a fixed relationship, and it may be reasonable to consider that their dominant wavelengths are the same.

When this transition tendency of the chromaticity towards the periphery was concerned with the results shown in Fig. 1D, which were plotted with Y's, there appeared a relationship that the $\mathrm{Y}^{\prime}$ values of the low-salinity group were always smaller than those of the highsalinity group. When this relationship was taken into consideration, the color of the middle portion which was reacted by the von Kossa's method likely shifted from dark achromatic color (thick black) to a more bright reddish color with the acclimatization to the environment of high-salinity.

With these results, it is estimated that the high-salinity environment reduced the amount of calcium inside of the crayfish aesthetasc hairs. This will coincide to the observations that the aesthetasc hairs of Japanese lobster have no accumulation of calcium (unpublished data).

With these observations, the calcium localization within the crayfish aesthetasc hairs is likely to serve as a factor to protect the receptor site of the sensory neurons innervating aesthetasc hairs from the external environment of low-salinity.

Acknowledgments. I am grateful to Dr. Yasuji Katsuki, M. J. A., for the continuous encouragement in preparing manuscripts, and to Dr. Kozo Okamoto, M. J. A., for his comments on the staining methods.

\section{References}

1) Chichibu, S. (1975) : Proc. XIV Annual Meeting, The Japan Society for Biophysics, 294 (in Japanese).

2) Chichibu, S., and Ishiguro, M. (1978): Acta med. Kinki Univ., 3, 81.

3) Chichibu, S., Wada, T., Komiya, H., and Suzuki, K. (1978) : Acta med. Kinki Univ. (in press).

4) Debaisieux, P. (1949): Cellule, 52, 311.

5) Gatsoff, P. S. (1959) : Culture Methods for Invertebrate Animals (eds. J. G. Needam et al.). Dover Publ. Inc., N.Y., p. 5 .

6) Laverack, M. S. (1964): Comp. Biochem. Physiol., 13, 301.

7) Laverack, M. S., and Ardill, D. J. (1965): Quart. J. micr. Sci., 106, 45.

8) Okamoto, K., and Maeda, R. (1976): Microscopic Histochemistry, 4th ed., vol. 1 (eds. Okamoto et al.), Igakushoin, Ltd., Tokyo, p. 33 (in Japanese).

9) Tazaki, K., and Shigenaga, T. (1974) : Comp. Biochem. Physiol., 47A, 195.

10) Thomas, W. J. (1970) : J. Zool., Lond., 160, 91. 